Macedonian Pharmaceutical Bulletin, 66 (Suppl 1) 83 - 84 (2020)

Online ISSN 1857 - 8969

UDC: $615.322: 614.253 .8$

DOI: 10.33320/maced.pharm.bull.2020.66.03.041

Short communication

\title{
Patients' knowledge and awareness of herbal medicines efficacy and safety in hospital settings
}

\author{
Sanja Filkova ${ }^{1} *$ Branka Pashaliska Cvetkov $^{2}$, Jasminka Patcheva $^{3}$, Sava Pejkovska ${ }^{4}$, \\ Olivera Krstic Nakovska ${ }^{4}$, Dimitar Karkinski ${ }^{4}$ \\ ${ }^{1}$ Public institution for the needs of University Clinics, Institutes and Urgent Centre, \\ Department of Clinical Pharmacy, Majka Tereza 17, 1000 Skopje, Republic of North Macedonia \\ ${ }^{2}$ University Clinic of Neurology, Majka Tereza 17, 1000 Skopje, Republic of North Macedonia \\ ${ }^{3}$ Pharmaceutical Chamber of Macedonia, 50 Divizija 34, 1000 Skopje, Republic of North Macedonia \\ ${ }^{4}$ University Clinic of Pulmology and Allergology, Majka Tereza 17, 1000 Skopje, Republic of North Macedonia
}

\section{Introduction}

Herbal medicines are one type of dietary supplements and people use them to try to maintain or improve their health and well-being. Many people believe that products labeled "natural" are always safe and good for them. This is not necessarily true. Herbal medicine probably presents a greater risk of adverse effects and interactions than any other complementary therapy (Vickers et al., 2001). Some dietary supplements have the potential for substantial hazard. These hazards raise issues about the regulatory and practical distinction between dietary supplements and drugs (Palmer et al., 2003). In addition, there is an ongoing problem with unexpected toxicity of herbal products due to quality issues, including use of poor quality herbal material, incorrect or misidentified herbs, incorrect processing methods, supply of adulterated or contaminated herbs or products (Shaw, 2010). Adverse reactions due to interactions may not be recognized if the physician or other health professional is not aware of the concomitant use of medicinal herbs (Giveon et al., 2004). Increased symptom severity was associated with use of several ingredients, long-term use and age. Associations between adverse events and ingredients are difficult to verify if a product has more than one ingredient, and because of incomplete information systems (Palmer et al., 2003). Vickers et al. (2001) have shown that "Serious adverse effects after administration of herbal products have been reported, and in most cases, the herbs involved were self-prescribed and bought over the counter or were obtained from a source other than a registered practitioner". Overlap of some pharmacological profiles further blurs the boundary between prescription pharmaceuticals and dietary supplements (Palmer and Howland, 2001). The role of the clinical pharmacist as an integral part of modern health care in hospital settings in terms of use of herbal medicines and dietary supplements is very significant. There are relatively few data regarding the use of herbal products in hospitalized patients and the incidence of such events remains unknown. The aim of this study is to determine the level of use of herbal products and other dietary supplements, evaluate and increase patients' knowledge and awareness of supplements efficacy and safety, and to explore whether there is a need to document the use of herbal products in the hospital settings.

*s_filkova@hotmail.com 


\section{Materials and methods}

This survey presents and discusses results obtained from analyzing responses to an anonymous questionnaire collected from patients receiving multiple sclerosis treatment at the University Clinic of Neurology in Skopje, North Macedonia. The questionnaire consisted of 14 questions. It contained demographic data such as gender, age, education level and profession and 10 questions related to the patients' use and knowledge of herbal medicines and exchanging information with medical staff about them. The survey was performed from December 2019 to February 2020. The obtained data were documented and descriptively analyzed.

\section{Results and discussion}

A survey was conducted among 36 patients with multiple sclerosis, 16 females and 20 males. The patients were between the ages of 38 and 73 years and they come from various North Macedonian cities. $72 \%$ of the respondents used herbal medicines and other dietary supplements before hospitalization; $44 \%$ reported to have either enough or a lot of knowledge of dietary supplements, while $56 \%$ knew little or nothing. Most often, they used Vitamin D3, Vitamin B, Vitamin C, Vitamin E, cannabis oil, aloe vera gel, garlic, St John's wort, valerian and herbal mixture. Family and friends were the main influence that determined use in $77 \%$ of the respondents, whereas a physician's or a pharmacist's referral was reported in $13 \%$, whereas media account for $7 \%$ of the cases. Only $23 \%$ of patients considered that herbal medicines can cause side effects, and $27 \%$ were aware of the possibility of interactions with conventional drugs. On admission to the hospital, $81 \%$ of the patients were not asked by the doctor if they used herbal medicines or other dietary supplements, while $89 \%$ of the patients considered that it was necessary to inform the doctor about it. There was no significant difference in provided answers based on demographic factors, level of education and profession with patient knowledge.

\section{Conclusion}

These findings confirm that a majority of patients used herbal medicines and vitamins without being asked about it by a physician or without informing him on their own initiative. They are still not aware of the risks to their health, the reasons for missing therapeutic efficacy, adverse effects and interactions with other conventional drugs and supplements. The challenge remains to improve patients' knowledge and awareness of herbal medicines and dietary supplements and to indicate the possible harmful consequences of the selfprescribed use. It is necessary to change the attitude of patients and medical staff to these products in order to prevent side effects and increase safety. The health care staff needs to know about the use of any herbal medicines, pharmacotherapy anamnesis on hospitalization should include this information, and the clinical pharmacist should assess the overall therapy and make the right decisions. Overall, further research into hazards and risks of herbal medicines and other dietary supplements use in hospitals should be a priority.

\section{References}

Giveon, S.M., Liberman, N., Klang, S, Kahan, E., 2004. Are people who use natural drugs aware of their potentially harmful side effects and reporting to family physician. Patient Educ. Couns. 53(1), 5-11. Available at: https://doi.org/10.1016/S07383991(03)00241-6.

Palmer, E.M., Howland, M.A., 2001. Herbals and dietary supplements, in: Ford, M., Delaney, K., Ling, L., Erickson, T. (Eds.), Clinical toxicology. WB Saunders, Philadelphia, pp. 316-331.

Palmer, E.M., Haller, C., McKinney, E.P., Schwartz, K.W., Tschirgi, A., Smolinske, C.S., Woolf, A., Sprague, M.B., Ko, R., Everson, G., Nelson, S.L., Butera, D.T., Bartlett, W.D., Landzberg, R.L., 2003. Adverse events associated with dietary supplements: an observational study. The Lancet 361(9352), 101106. Available at: https://doi.org/10.1016/S01406736(3)12227-1.

Shaw, D., 2010, Toxicological risks of Chinese herbs. Planta Med. 76(17), 2012-2018. Available at: https://doi.org/10.1055/S0030-1250533.

Vickers, A., Zollman, C., Lee, R., 2001. Herbal medicine. West J Med. Available at: https://doi.org/10.1136/ewim.175(2)125.

Maced. Pharm. Bull. 66 (Suppl 1) 83 - 84 (2020) 